\title{
INDUÇÃO DE MÚLTIPLAS OVULAÇÕES EM ÉGUAS COM BAIXAS DOSES DE ACETATO DE DESLORELINA SEM ACOMPANHAMENTO FOLICULAR PRÉVIO
}

Lorenzo Garrido Segabinazzi ${ }^{1}$, Luiz Felipe Steigleder ${ }^{2}$, Rodrigo Kaipper ${ }^{2}$, Teodoro Silva Rodrigues ${ }^{2}$, Otávio Aragão ${ }^{2}$, José Antonio Dell'Aqua Jr. ${ }^{1}$, Marco Antonio Alvarenga $^{1}$

1'Departamento de Reprodução Animal e Radiologia Veterinária, Universidade Estadual Paulista "Júlio de Mesquita Filho" Unesp - Botucatu, SP, Brasil lgseg@hotmail.com

${ }^{2}$ Coudelaria de Rincão e Campo de Instrução de Rincão, Exército Brasileiro - São Borja, RS, Brasil.

Recebido em: 08/09/2015 - Aprovado em: 14/11/2015 - Publicado em: 01/12/2015 DOI: http://dx.doi.org/10.18677/Enciclopedia_Biosfera_2015_177

O objetivo deste estudo foi avaliar a possibilidade de induzir múltiplas ovulações em éguas com baixas doses de acetato de deslorelina sem controle folicular prévio. Foram utilizadas 11 éguas que responderam ao protocolo de sincronização de cio com PGF2 $\alpha$ aplicada duas vezes em intervalo de 14 dias. No ciclo tratado (CT), após 48 horas da segunda aplicação de PGF2 $\alpha$ iniciou-se o protocolo para indução de múltiplas ovulações utilizando $125 \mu \mathrm{g}$ de acetato de deslorelina por via IM, a cada 12 horas durante três dias consecutivos. No terceiro dia iniciou-se a avaliação ultrassonográfica dos animais e a indução da ovulação foi realizada com $750 \mu \mathrm{g}$ de acetato de deslorelina e $1650 \mathrm{UI}$ de hCG, quando dois ou mais folículos $\geq 35 \mathrm{~mm}$ ou um folículo $\geq 38 \mathrm{~mm}$ foram observados. Foram considerados como ciclos controles, dados de dois ciclos anteriores ao início do experimento, um ciclo controle positivo (CP) (indução de duplas ovulações com acompanhamento folicular) e um ciclo negativo (CN) sem tratamento. As variáveis foram analisadas através do Teste t. $\mathrm{O}$ número de folículos pré-ovulatorios (CT - 19; CP - 22; CN - 13), ovulações (CT 18; CP - 22; CN - 13) e ovulações/ciclo (CT - 1,72; CP - 2; CN - 1,18) foram maiores no ciclo tratado e controle positivo quando comparado ao ciclo controle negativo. Com base nos resultados deste estudo, o protocolo proposto foi eficiente em elevar o número de ovulações em éguas tratadas com baixas doses de acetato de deslorelina, mesmo sem controle folicular prévio ao início do tratamento.

PALAVRAS-CHAVE: crescimento folicular, Egua, $\mathrm{GnRH}$, recuperação embrionária, transferência de embriões

\section{INDUCTION OF MULTIPLE OVULATIONS IN MARES USING DESLORELIN ACETATE WITHOUT PRIOR FOLLICULAR MONITORATION}

\begin{abstract}
The present study aimed to evaluate the possibility of induction multiple ovulations in mares with deslorelin acetate without the prior follicular control. Eleven mares that responded to the estrus synchronization protocol with two doses of PGF2 $\alpha$ with interval of 14 days were used. In treated cycle (TC), 48 hours after the second
\end{abstract}


PGF2 $\alpha$ application, the protocol was initiated using injections of $125 \mu \mathrm{g}$ of deslorelin acetate every 12 hours for three consecutive days. On the third day, the mares were daily evaluated by ultrasonography, and induction of ovulation was done with $750 \mu \mathrm{g}$ of deslorelin acetate and 1650 IU of hCG when two or more follicles $\geq 35 \mathrm{~mm}$ or a follicle $\geq 38 \mathrm{~mm}$ were observed. Were considered controls cycles, data from two previous cycles to the beginning of the experiment, a positive control cycle (PC) (induction of double ovulations with follicular monitoring) and a negative cycle (NC) which had no treatment for induction of multiple ovulations. The variables studied compared using Student's t-test. The number of pre-ovulatory follicles (TC - 19; PC 22; NC - 13), ovulation (TC - 18; PC - 22; NC - 13) and ovulations/cycle (TC - 1.72; PC - 2; NC - 1.18) were higher in treated cycle and in positive control cycle when compared to the negative control cycle. Based on the results of this study, the proposed protocol was effective into increase the number of ovulations in treated mares with low doses of deslorelina acetate, even without prior follicular monitoration.

KEYWORDS: Mare, embryo transfer, GnRH, embryo recovery, follicular growth.

\section{INTRODUÇÃO}

Com a expansão da equinocultura nas últimas décadas, os criadores têm procurado otimizar a eficiência reprodutiva dos seus animais, buscando incrementar sua produção através de um maior número de embriões coletados por égua doadora. A transferência de embriões tem sido utilizada como uma biotecnologia da reprodução animal para produção de múltiplos produtos de éguas que possuem alto valor genético em uma mesma estação de monta, elevando a eficiência reprodutiva desses animais (HURTGEN, 2008).

A égua é um animal monovulatório e a possibilidade de incrementar o número de ovulações por ciclo traz benefícios aos programas de TE, já que as taxas médias de recuperação embrionária e de prenhez nessa espécie são de 60\% (ALVARENGA et al., 2008), quando a colheita provem de ovulações únicas e induzidas. Por este motivo estima-se que sejam necessários em média três ciclos por doadora para garantir uma prenhez.

Para melhorar estes índices, a utilização de tratamentos superovulatórios se apresenta como uma alternativa, uma vez que estes tratamentos visam à obtenção de mais de uma ovulação e consequentemente aumentam as chances de obter-se pelo menos um embrião por ciclo da égua (SQUIRES et al. 2003). Assim, a superovulação é uma importante ferramenta para o uso rotineiro na TE em equinos, a fim de reduzir os custos e melhorar a eficiência dos programas nesta espécie (ALVARENGA et al., 2008).

Apesar de a superovulação ser uma técnica aplicável em equinos, com a utilização de FSH equino purificado (e-FSH), extrato de pituitária equina (EPE) e FSH recombinante (re-FSH), aumentando o número de folículos pré-ovulatorios e ovulações (ALVARENGA et al., 2001, LOGAN et al., 2007, RAZ et al., 2009, CULLINGFORD et al. 2010, MEYERS-BROWN et al. 2010, MCKKINON et al. 2011, MEYERS-BROWN et al. 2011) as taxas de recuperação embrionária são baixas em relação ao número de ovulações/ciclo com 0 uso destes tratamentos. Isto provavelmente ocorre devido à formação de um grande coágulo na fossa de ovulação após as primeiras ovulações, impedindo o transporte e captura dos oócitos liberados subsequentemente até a tuba uterina (CARMO et al., 2006). Este fato é explicado pelas particularidades do ovário das éguas, que comparado às outras 
espécies tem um maior tamanho e o posicionamento invertido das camadas cortical e medular, desse modo obrigando a ocorrência de todas as ovulações através da fossa de ovulação (HYRANO et al., 2009).

Recentemente NAGAO et al. (2012), demonstraram que o uso de baixas doses de acetato de deslorelina, próximo ao momento da dominância folicular, induzem duplas ovulações em éguas e melhoram as taxas de recuperação embrionária. Entretanto para este tratamento ser efetivo, é necessário realizar um acompanhamento folicular prévio das éguas e iniciar o tratamento no momento adequado, necessitando assim de um acompanhamento diário por ultrassonografia, o que não se torna prático à campo.

Desta forma, o presente estudo, teve como objetivo verificar a eficiência da utilização de baixas doses de deslorelina para indução de múltiplas ovulações, sem o acompanhamento folicular prévio ao início do tratamento.

\section{MATERIAL E MÉTODOS}

Este estudo foi aprovado pela comissão de ética no uso de animais da Faculdade de Medicina Veterinária e Zootecnia - UNESP/Botucatu, sob o número de protocolo 11/2009.

Inicialmente 18 éguas da raça Brasileiro de Hipismo, com idade entre três e 19 anos, pesando entre 400 e 600 kg, pertencentes à Coudelaria de Rincão (Haras do Exército Brasileiro), localizado em São Borja - RS/Brasil (latitude 2844'27 .6 "S e longitude 5535'05 .0" O) foram utilizadas. Todas a s éguas receberam previamente duas aplicações de 5mg de dinoprost tromethamina - PGF2a (Lutalyse®, RhodiaMérieux, Brasil) com intervalo de 14 dias, sem prévia avaliação quanto ao estágio estral desses animais.

O protocolo para a indução de múltiplas ovulações foi iniciado 48 horas após a segunda aplicação de PGF2 $\alpha$, sem qualquer avaliação do status reprodutivo das éguas e consistia na aplicação de $125 \mu \mathrm{g}$ por via intramuscular (IM) de acetato de deslorelina (Sincrorrelin $\AA$ - Ouro Fino, Brasil) a cada 12 horas. No terceiro e quarto dia de tratamento com acetato de deslorelina as éguas foram avaliada através de palpação retal e ultrassonografia e as que não responderam a sincronização de cio, ou seja, não apresentaram nenhum folículo $\geq 28 \mathrm{~mm}$ foram descartadas (7 éguas).

Nos animais que apresentaram no mínimo um folículo $\geq 28 \mathrm{~mm}$ durante estas avaliações (11 éguas), o tratamento com acetato de deslorelina foi continuado até a detecção de dois ou mais folículos $\geq 35 \mathrm{~mm}$ ou um $\geq 38 \mathrm{~mm}$ e edema endometrial grau 2 (SAMPER, 2008), quando a ovulação foi induzida utilizando $750 \mu \mathrm{g}$ de acetato de deslorelina IM e 1650 UI de Gonadotropina Coriônica Humana (hCG, Vetecortß Hertape Calier, Brasil) por via intravenosa (IV) e este foi considerado o ciclo tratado.

Como grupos controle, foram avaliados dois ciclos de cada animal, anteriores ao início do experimento, referente às taxas de ovulação e número de folículos préovulatórios, onde em um as éguas não foram submetidas a tratamento superovulatório e apenas feito acompanhamento diário dos animais através de palpação retal e ultrassonografia (Ciclo Controle Negativo) e outro onde os animais foram submetidos a um protocolo de indução de múltiplas ovulações descrito por NAGAO et al. (2012), com controle folicular prévio e início do tratamento utilizando $125 \mu \mathrm{g}$ de acetato de deslorelina a cada 12 horas quando observado dois folículos com tamanho entre 20 e $25 \mathrm{~mm}$ de diâmetro e indução da ovulação realizada como descrito anteriormente (Ciclo Controle Positivo). No ciclo controle negativo, a indução da ovulação foi realizada com $750 \mu \mathrm{g}$ de acetato de deslorelina quando observado um folículo $\geq 35 \mathrm{~mm}$ e edema uterino grau 2 (SAMPER, 2008). 
Para todas as análises foram calculadas as médias e desvio padrão e então realizado 0 teste de normalidade Kolmogorov-Smirnov. Como os dados apresentavam distribuição normal, utilizou-se o teste $\mathrm{t}$ pareado. $\mathrm{O}$ valor de significância considerado foi de $5 \%$. As análises foram realizadas pelo programa GraphPad Prism versão 5.0 para Windows (GraphPad software, San Diego, Califórnia, EUA).

\section{RESULTADOS E DISCUSSÃO}

No protocolo proposto no presente estudo, propositalmente as éguas não foram avaliadas quanto a atividade ovariana antes do início do estudo, e pode-se observar que apenas $61 \%$ das éguas responderam a sincronização de cio, o que ocorreu possivelmente pelo fato de este estudo ter sido realizado no final da estação reprodutiva no Hemisfério Sul e algumas éguas já estariam em fase de transição de outono.

No entanto, já nas éguas que responderam a aplicação de PGF2a, foi possível observar maior número de folículos pré-ovulatorios $(p<0,05)$ em comparação ao ciclo controle negativo desses animais e não foi observada diferença $(p>0,05)$ em relação ao ciclo controle positivo como demonstrado na Tabela 1. No ciclo tratado oito éguas $(72,7 \%)$ exibiram crescimento de mais de um folículo préovulatório, sete destas $(63,6 \%)$ apresentaram dois folículos com tamanho $\geq 35 \mathrm{~mm}$ e uma $(9,1 \%)$ três, resultado semelhante ao do grupo controle positivo, onde nove éguas $(81,8 \%)$ apresentaram dois folículos pré-ovulatórios e uma $(9,1 \%)$ três. Já no controle negativo apenas duas $(18,2 \%)$ apresentaram mais de um folículo préovulatório. Isso ocorre pois os análogos do $\mathrm{GnRH}$ estimulam a secreção de gonadotrofinas na hipófise, aumentando a secreção endógena de FSH e LH. Este aumento de FSH promove o crescimento folicular, pois a hipófise anterior responde a aplicações exógenas de GnRH (GINTHER, 2000), não permitindo que aconteça o início da divergência folicular, ocorrendo assim o crescimento dos dois maiores folículos presentes nos ovários.

O número de ovulações e ovulações/ciclo também foi maior $(p<0,05)$ no ciclo tratado (Tabela 1) em relação ao ciclo controle negativo, mas não diferiu do controle positivo. Foram observadas ovulações em todos os folículos pré-ovulatórios nos grupos controle positivo e negativo, apenas uma égua $(9,1 \%)$ no grupo tratado ovulou um folículo e apresentou um folículo hemorrágico anovulatório. Estes resultados corroboram com os achados de NAGAO et al. (2012), que utilizaram este protocolo com controle folicular prévio, iniciando antes do estabelecimento da dominância folicular, quando havia ao menos dois folículos nos ovários com tamanho entre 20 e $25 \mathrm{~mm}$, o que ocorria em média 2,3 dias após a aplicação de PGF2 $\alpha$, impedindo desse modo a regressão do folículo co-dominante em $82 \%$ das éguas. Isso explica o fato de uma das éguas do nosso experimento que apresentou dupla ovulação no ciclo controle, apresentar ovulação tripla quando tratada com acetato de deslorelina.

O tempo entre a aplicação de PGF2 $\alpha$ e a indução da ovulação também diferiu $(p<0,05)$ entre os grupos como podemos observar na Tabela 1 , sendo este diminuído quando utilizado o acetato de deslorelina para estimulação do crescimento folicular. Resultado semelhante ao descrito por NAGAO et al. (2012). 
TABELA 1. Médias e desvio padrão das variáveis analisadas entre os ciclos controle e o ciclo tratado com acetato de deslorelina para indução de múltiplas ovulações sem controle folicular prévio.

\begin{tabular}{|c|c|c|c|}
\hline Variáveis & $\begin{array}{l}\text { Controle } \\
\text { Negativo }\end{array}$ & $\begin{array}{l}\text { Controle } \\
\text { Positivo }\end{array}$ & Tratado \\
\hline $\begin{array}{l}\text { Número de folículos } \\
\text { pré-ovulatórios }\end{array}$ & $13 \pm 0,4^{a}$ & $22 \pm 0,4^{b}$ & $19 \pm 0,6^{b}$ \\
\hline Número de ovulações & $13 \pm 0,4^{\mathrm{a}}$ & $22 \pm 0,4^{b}$ & $18 \pm 0,7^{b}$ \\
\hline $\begin{array}{ll}\text { Número } & \text { de } \\
\text { ovulacões/ciclo }\end{array}$ & $1,18^{\mathrm{a}}$ & $2^{b}$ & $1,72^{\mathrm{b}}$ \\
\hline $\begin{array}{l}\text { Número de éguas com } \\
\text { mais de um folículo } \\
\text { pré-ovulatório }\end{array}$ & $2(18 \%)^{a}$ & $10(91 \%)^{b}$ & $8(73 \%)^{b}$ \\
\hline $\begin{array}{l}\text { Intervalo (dias) PGF2a } \\
\text { até a indução da } \\
\text { ovulação }\end{array}$ & $7 \pm 2,6^{\underline{a}}$ & $3 \pm 0,6^{b}$ & $3,5 \pm 1,0^{b}$ \\
\hline
\end{tabular}

A duração do tratamento com acetato de deslorelina também é um fator importante pela possibilidade de ocorrência do fenômeno "down regulation", que ocorre por aplicações excessivas e contínuas de grandes doses de $\mathrm{GnRH}$, o qual inicialmente induz a uma hipersecreção de gonadotrofinas e posteriormente a uma dessensibilização da glândula pituitária, promovendo assim a redução na liberação de gonadotrofinas (IRVINE \& ALEXANDRE, 1993). Isso foi observado por NAGAO et al. (2012) em seu estudo, quando iniciou o tratamento com acetato de deslorelina $(100 \mu \mathrm{g})$ imediatamente após a indução da luteólise com $5 \mathrm{mg}$ de dinoprost tromethamina (Lutalyse $\AA^{\circledR}$, Pfizer, New York, NY, USA) e em três éguas $(37,5 \%)$ onde o tratamento durou 7 dias, houve uma parada no crescimento folicular após o quarto dia da aplicação de deslorelina e após 3 dias regressão destes folículos, não evoluindo para ovulação. GIMENES et al. (2012) em um estudo utilizando extrato de pituitária equina quando reduziu a duração do protocolo, iniciando o tratamento após a detecção de folículos com tamanho entre 20 e $23 \mathrm{~mm}$, obteve sucesso na indução de múltiplas ovulações, corroborando com os resultados do presente estudo.

Quando comparadas as ovulações múltiplas não houve diferença $(p>0,05)$ no número de ovulações unilaterais e bilaterais (Tabela 2), corroborando com os achados de RIERA et al. (2006), entretanto, discordando de DUARTE (2003) e NAGAO (2012) que verificaram uma maior ocorrência de ovulações unilaterais em éguas com ovulações múltiplas. 
TABELA 2. Número de ovulações bilaterais e ovulações unilaterais quando analisadas as ovulações múltiplas.

\begin{tabular}{lcc}
\hline Grupo & $\begin{array}{c}\text { Número de ovulações } \\
\text { bilaterais }\end{array}$ & $\begin{array}{c}\text { Número de ovulações } \\
\text { unilaterais }\end{array}$ \\
\hline Controle Negativo & 1 & 1 \\
Controle Positivo & 5 & 5 \\
Tratado & 3 & 4 \\
\hline
\end{tabular}

\section{CONCLUSÃO}

Com base nos resultados deste estudo, conclui-se que o presente protocolo foi eficiente em aumentar o número de ovulações por ciclo, sendo de mais fácil aplicabilidade a campo pela necessidade de um monitoramento menos frequente das éguas doadoras de embriões.

\section{REFERÊNCIAS}

ALVARENGA, M. A.; MCCUE, P. M.; BRUEMMER, J.; NEVES NETO, J. R.; SQUIRES, E. L. Ovarian superstimulatory response and embryo production in mares treated with equine pituitary extract twice daily. Theriogenology, v. 56, p. 879-887, 2001.

ALVARENGA, M. A.; CARMO, M. T.; LANDIM-ALVARENGA, F. C. Superovulations in mares: limitations and perspectives. Pferdeheilkunde, v. 24, p. 88-91, 2008.

CARMO, M. T.; LOSINO, L.; AQUILAR, J. J.; ARAUJO, G. H. M.; ALVARENGA, M. A. Oocyte transport to the oviduct of superovulated mares. Animal Reproduction Science, v. 94, p. 337-339, 2006.

CULLINGFORD, E. L.; SQUIRES, E. L.; MCCUE, P. M.; SEIDEL Jr, G. E. Attempts at superovulation of mares with porcine follicle stimulating hormone and recombinant equine follicle stimulating hormone. Journal of Equine Veterinary Science, v. 30, p. 305-309, 2010.

DUARTE, M. B. Incidência de duplas ovulações, prenhez gemelar e eficácia da redução manual de gêmeos em éguas Quarto de Milha. Dissertação (Mestrado). Universidade Federal de Uberaba, Uberaba/MG, 2003.

GIMENES, A. M.; IGNÁCIO, F. S.; BOFF, A. L. N.; BERGFELT, D. R.; MEIRA, C. Enhanced ovarian response to low-dose treatment with equine pituitary extract in mares. Reproduction Fertility and Development, v. 22, p. 362, 2012.

GINTHER, O. J. Selection of the dominant follicle in cattle and horses. Animal Reproduction Science, v. 60, p. 61-79, 2000.

HIRANO, Y.; KIMURA, J.; NAMBO, Y.; YOKOTA, H.; NAKAMURA, S.; TAKEMOTO, S.; HIMENO, R.; MISHIMA, T.; MATSUI, M.; MIYAKE, Y.I. Population of follicles and luteal structures during the oestrous cycle of mares detected by three-dimensional internal structure microscopy. Anat. Histol. Embryol, v. 38, p. 214-21, 2009. 
HURTGEN, J.P. Management of embryo donor mares with chronic infertility. Proceedings 54th Annual Convention of the American Association of Equine Practitioners, San Diego, California, p. 414-417, 2008.

IRVINE, C. H. G. AND ALEXANDER, S. L. GnRH. In: Equine Reproduction. Malvern: Lea \& Febiger, p. 37-44, 1993.

LOGAN, N. L.; MCCUE, P. M.; ALONSO, M. A.; SQUIRES, E. L. Evaluation of three equine FSH superovulation protocols in mares. Animal Reproduction Science, v. 102, p. 48-55, 2007.

MCKINNON, A. O. Origin and outcome of twin pregnancies. In: McKinnon, A. O., Squires, E. L., Vaala, W.E., Varner, D.D., editors. Equine reproduction. Second edition, v. 2. Oxford: Wiley-Blackwell; p. 2350-2358, 2011.

MEYERS-BROWN, G. A.; MCCUE, P. M.; NISWENDER, K. D.; SQUIRES, E. L.; DELUCA, C. A.; BIDSTRUP, L. A.; COLGIN, M.; FAMULA, T. R.; ROSER, J. F. Superovulation in mares using recombinant equine follicle stimulation hormone: ovulation rates, embryo retrieval, and hormone profiles. Journal of Equine Veterinary Science, v. 30, p. 560-568, 2010.

MEYERS-BROWN, G.; BIDSTRUP, L. A.; FAMULA, T. R.; COLGIN, M.; ROSER, J. $\mathrm{F}$. Treatment with recombinant equine follicle stimulating hormone (reFSH) followed by recombinant equine luteinizing hormone (reLH) increases embryo recovery in superovulated mares. Animal Reproduction Science, v. 128, p. 52-59, 2011.

NAGAO, J. F.; NEVES NETO, J. F.; PAPA, F. O.; ALVARENGA, M. A.; DELL'AQUA, C. P.; DELL'AQUA Jr., J. A. Induction of double ovulation in mares using deslorrelin acetate. Animal Reproduction Science, v. 136, p. 69-73, 2012.

RAZ, T.; CARLEY, S.; CARD, C. Comparison of the effects of eFSH and deslorelin treatment regimes on ovarian stimulation and embryo production of donor mares in early vernal transition. Theriogenology, v. 71, p. 1358-1366, 2009.

RIERA, F. L.; ROLDÁN, J. E.; HINRICHS, K. Patterns of embryo recovery in mares with unilateral and bilateral double ovulations. Animal Reproduction Science, v. 94, p. 398-399, 2006.

SAMPER, J. C. Induction of estrous and ovulation: Why some mares respond and others do not. Theriogenology, v. 70, p. 445-447, 2008.

SQUIRES, E. L.; CARNEVALE, E. M.; MCCUE, P. M.; BRUEMMER, J. E. Embryo technologies in the horse. Theriogenology, v. 59, p. 151-170, 2003. 